\title{
An interpolation between the Wave and Diffusion equa- tions through the Fractional Evolution equations Dirac like
}

\author{
T. Pierantozzi ${ }^{\mathbf{a}}$ and L. Vázquez ${ }^{\mathbf{a}, \mathbf{b}}$ \\ a Departamento de Matemática Aplicada, Facultad de Informática, \\ Universidad Complutense de Madrid, Madrid, E-28040 Spain \\ Teresa_Pierantozzi@mat.ucm.es \\ b Centro de Astrobiología (CSIC-INTA), \\ Torrejón de Ardoz, Madrid, 28850 Spain \\ Ivazquez@fdi.ucm.es
}

(Received: )

Through the Fractional Calculus and following the method used by Dirac to obtain his well-known equation from the Klein-Gordon equation, we analyze a possible interpolation between the Dirac and the diffusion equations in one space dimension. We study the transition between the hyperbolic and parabolic behaviors by means of the generalization of the D'Alembert formula for the classical wave equation and the invariance under space and time inversions of the interpolating Fractional Evolution equations Dirac like. Such invariance depends on the values of the fractional index and it is related to the non local property of the time fractional differential operator. For this system of fractional evolution equations, we also find an associated conserved quantity analogous of the Hamiltonian for the classical Dirac case.

PACS: 02.30.Gp, 02.50.-r, 03.65.Pm, 44.05.+e, 45.10.Hj.

Keywords: fractional differential equations, Riemann-Liouville fractional integrals and derivatives, Caputo fractional derivative, Mittag-Leffler and Wright functions, Diractype equations.

\section{Introduction}

Following the well known Dirac's approach [19], the free Dirac equation can be considered as the square root of the Klein-Gordon equation. In a more general context Morinaga and Nono [11] analyzed the integer s-root of the partial differential equations of the form

$$
\sum_{|I|=s} a_{I} \frac{\partial^{|I|}}{\partial x^{I}} \phi=\phi,
$$

by defining them as the first order system

$$
\sum_{i=1}^{n} \alpha_{i} \frac{\partial \Phi}{\partial x_{i}}=\Phi
$$

where $\alpha_{1}, \ldots \alpha_{n}$ are matrices. From the physical point of view the $\alpha_{k}$ describe internal degrees of freedom of the associated system. 
In the above context, Vázquez et al. recently considered in [21] and [22] the fractional diffusion equations with internal degrees of freedom. They can be obtained as the s-roots of the standard scalar linear diffusion equation. Thus, a possible definition of the square root of the standard diffusion equation ( $\mathrm{SDE}$ ) in one space dimension, $u_{t}-u_{x x}=0$, is the following:

$$
\left(A \frac{\partial^{1 / 2}}{\partial t^{1 / 2}}+B \frac{\partial}{\partial x}\right) \psi(x, t)=0
$$

where $A$ and $B$ are $2 \times 2$ matrices satisfying Pauli's algebra

$$
A^{2}=I, \quad B^{2}=-I, \quad A B+B A=0,
$$

being $I$ the identity operator.

It is worthy of mention that Oldham and Spanier [12] were the first authors to derive a formulation involving the mathematical operation of semidifferentiation in replacement of the Fick's laws in a work of 1970.

Here $\psi(x, t)$ is a multicomponent function with at least two scalar space-time components. Also, each scalar component satisfies the SDE. Such solutions can be interpreted as probability distributions with internal structure associated to internal degrees of freedom of the system. They are named diffunors in analogy with the spinors in Quantum Mechanics.

In this paper we deal with a further generalization of Dirac's method, considering the system of fractional evolution equations

$$
\left(A \partial_{t}^{\alpha}+B \partial_{x}\right) \psi(t, x)=0, \quad \psi(t, x)=\left(\begin{array}{c}
u_{1}(t, x) \\
u_{2}(t, x)
\end{array}\right),
$$

with $0<\alpha<1, A$ and $B$ satisfying (4), as the square root of the time Fractional Diffusion-Wave Equation

$$
\partial_{t}^{2 \alpha} u(t, x)-\partial_{x x} u(t, x)=0 .
$$

Equation (6), associated to anomalous diffusion, has been widely studied in the literature by many authors, among which Schneider and Wyss [18], Metzler et al. ([7], [9], [23]), Mainardi et al. [6], [5], [4], Sokolov et al. [20], Saichev et al. [16]. A more physical discussion of this equation was given by Metzler and Klafter in [8] and it is worthwhile mentioning that they proved that the resulting probability density is bimodal in character. In this sense, Schneider and Wyss [18] showed that, for dimensions higher than 1, the character of the solution of the fractional wave equation (when $1 / 2<\alpha \leq 1$ ) as a proper probability density is lost.

Each component of $\psi(t, x)$ satisfies (6) while the index property $\partial_{t}{ }^{\alpha} \partial_{t}{ }^{\alpha} u=\partial_{t}{ }^{2 \alpha} u$ holds. Thus, in the interval $1 / 2<\alpha<1$, the decomposition (5) of (6), expressed in terms of fractional evolution equations of Dirac-type, represents a fractional interpolation between the diffusion $(\alpha=1 / 2)$ and wave $(\alpha=1)$ equations.

The applications of the fractional calculus range in a wide spectrum of areas like material sciences (viscoelasticity, polymers,...), circuits, diffusion processes, Biology, Economy, Geology, traffic problems, data analysis, and others, as it is illustrated, for example, in the text books by Hilfer [2], Ross [15], Samko et al. [17]. Many of the associated models amount to replace the time derivative in an evolution equation with a fractional derivative of real order. 
The fractional derivative operator $\partial_{t}^{\alpha}$ appearing in (5) can be specified according to several definitions available in the literature (see for example [17] and [14]); we will refer to the two commonly used definitions of Riemann-Liouville and Caputo.

The Riemann-Liouville derivative of order $\alpha>0$ is defined as

$$
\left({ }_{a}^{R L} D_{x}^{\alpha} f\right)(x)=\frac{d^{n}}{d x^{n}} \frac{1}{\Gamma(n-\alpha)} \int_{a}^{x}(x-t)^{n-\alpha-1} f(t) d t,
$$

with $x>a, n=-[-\alpha]$, whereas the Caputo fractional derivative, usually considered as a regularised version of the Riemann-Liouville fractional differential operator, takes the form

$$
\left({ }_{a}^{C} D_{x}^{\alpha} f\right)(x)=\frac{1}{\Gamma(n-\alpha)} \int_{a}^{x} \frac{f^{n}(\tau)}{(x-\tau)^{\alpha-n+1}} d \tau .
$$

There exists the following relation between the above definitions

$$
\left({ }_{a}^{C} D_{x}^{\alpha} f\right)(x)={ }_{a}^{R L} D_{x}^{\alpha}\left[f(x)-\sum_{j=0}^{n-1} f^{(j)}(a+) \frac{(x-a)^{j}}{j !}\right],
$$

and, a condition under which both derivatives hold is that $f \in A C^{n-1}(a, \infty)$. Equivalence (9) allows to use only initial conditions of the classical type when dealing with fractional equations involving Riemann-Liouville or Caputo derivatives. The integral approach pursued by Schneider and Wyss in [18] is probably the most physical way of incorporating these initial values to the corresponding equation.

Definitions (7) and (8) reproduce the classical derivative $\frac{d^{n}}{d x^{n}}$ when $\alpha=n(n \in \mathbb{N})$ and the identity operator for $\alpha=0$, and they are non local operators being given by a definite integral.

It is important to highlight that, in general (see [10] and [14], for example) the index property ${ }_{a} D_{x a}^{\alpha} D_{x}^{\beta} f={ }_{a} D_{x}^{\alpha+\beta} f$ does not hold using the definitions of fractional derivative of Riemann-Liouville (7) or Caputo (8), unless the function $f$ verifies:

$$
f^{(j)}(a+)=0, \quad j=0,1,2, \ldots, m-1,
$$

whenever $f(x) \in A C^{m-1}(a, \infty)$ and $f^{m}(x) \in L_{l o c}^{1}(a, \infty), m-1<\beta \leq m$.

In [10, Cap.4] it is analyzed a restricted class of functions $C$ for which the RiemannLiouville fractional derivative satisfies the cited index property. Some examples of functions in $C$ are: $x^{\lambda}$ with $\lambda>-1$, the polynomials, the exponentials, the sine and cosine functions and all the linear combinations of them.

The structure of the paper is as follows. In Sec. 2, a physical meaning to the solutions of $(5)$ is given, showing how they generalize the behavior of the solutions of the Dirac system, recovered when $\alpha=1$, in relation with the D'Alembert solution. Following the analogy with the classical Dirac case, the internal symmetries of the system (5) under inversions and translations in time and/or space, and Galileo transform are considered in Sec. 3, whereas in Sec. 4 a conserved quantity for (5), analogous of the Hamiltonian for the classical Dirac equation, is found. 


\section{Physical meaning of the solutions of the system of fractional evolution equations}

It is well known that the general solution of the wave equation with zero initial velocity,

$$
\left\{\begin{array}{c}
\partial_{t t} u(t, x)-c^{2} \partial_{x x} u(t, x)=0 \\
u(0, x)=\varphi(x), \quad \varphi \in \mathcal{C}^{2} \\
u_{t}(0, x)=0
\end{array}\right.
$$

is given by the D'Alembert Formula

$$
u(t, x)=\frac{1}{2}[\varphi(x-c t)+\varphi(x+c t)] .
$$

From a physical point of view, we can interpret this fact as that the amplitude at time $t$ of a perturbation created by a given starting deformation at rest, $\varphi(x)$, is the superposition of two waves, $\varphi(x+c t)$ and $\varphi(x-c t)$, whose shape is identical to the starting one's and travelling in opposite directions. The two waves are solutions of the following first order problems

$$
\left\{\begin{array}{c}
\partial_{t} u(t, x)-c \partial_{x} u(t, x)=0 \\
u(0, x)=\varphi(x), \quad \varphi \in \mathcal{C}^{2}
\end{array}\right.
$$

and

$$
\left\{\begin{array}{c}
\partial_{t} u(t, x)+c \partial_{x} u(t, x)=0 \\
u(0, x)=\varphi(x), \quad \varphi \in \mathcal{C}^{2}
\end{array}\right.
$$

and the D'Alembert solution is a linear combination of them with coefficients equals to $1 / 2$.

Now, if we only shrink the study to the pure real matrices leading to a system (5) of separated equations, then we have two possible choices:

$$
A=\left(\begin{array}{ll}
0 & 1 \\
1 & 0
\end{array}\right) \quad \text { y } \quad B=\left(\begin{array}{cc}
0 & 1 \\
-1 & 0
\end{array}\right)
$$

and the second pair of matrices given by the same $A$ and $-B$.

Substituting (15) in (5), it reduces to the following system of equations

$$
\left\{\begin{array}{c}
\partial_{t}^{\alpha} u_{1}(t, x)-\partial_{x} u_{1}(t, x)=0 \\
\partial_{t}^{\alpha} u_{2}(t, x)+\partial_{x} u_{2}(t, x)=0
\end{array},\right.
$$

where $0<\alpha<1$, and the equations appearing in (13) and (14) are devolved for the limiting case of $\alpha=1$ and $c=1$.

We want to show that the solution $u(t, x)$ of the Time Fractional Diffusion Equation (6) is still a linear combination of the solutions $u_{1}(t, x)$ and $u_{2}(t, x)$ of (16), for each

$0<\alpha<1$ and, therefore, that the relation existing between the Dirac solutions and the D'Alembert expression is extended to the fractional case.

In [3] the following initial value problem

$$
\begin{gathered}
\left({ }^{C} D_{t}^{\alpha} u\right)(t, x)=\lambda \partial_{x} u(t, x) \quad(t>0, x \in \mathbb{R} ; 0<\alpha<1), \\
\lim _{|x| \rightarrow \infty} u(t, x)=0, \quad u(0+, x)=g(x),
\end{gathered}
$$


has been solved and the general solution, expressed in terms of the inverse of its Fourier Transform, is given by

$$
u(t, x)=u_{g, \lambda}(t, x)=\frac{1}{2 \pi} \int_{-\infty}^{+\infty} E_{\alpha, 1}\left(-i \lambda k t^{\alpha}\right) G(k) e^{-i k x} d k,
$$

where $E_{\alpha, \beta}(z)$ is the biparametric Mittag-Leffler special function [1]. This solution is said to be localized due to the property $\lim _{|x| \rightarrow \infty} u(t, x)=0$.

On the other hand, the general localized solution of the Cauchy problem for the time fractional diffusion equation,

$$
\begin{gathered}
\left({ }^{C} D_{t}^{2 \alpha} f\right)(t, x)=\lambda^{2} \partial_{x x} f(t, x) \\
\lim _{|x| \rightarrow \infty} f(t, x)=0, \quad f(0+, x)=g(x), \quad\left[\partial_{t} f(t, x)\right]_{t=0}=0, \quad(t>0, x \in \mathbb{R}),
\end{gathered}
$$

where $0<\alpha<1$, can be found in [4] and it reads

$$
f(t, x)=f_{g}(t, x)=\frac{1}{2 \pi} \int_{-\infty}^{+\infty} E_{2 \alpha, 1}\left(-(\lambda k)^{2} t^{2 \alpha}\right) G(k) e^{-i k x} d k
$$

Now, if we apply the duplication formula [1] for the Mittag-Leffler function,

$$
E_{2 \alpha, 1}(z)=\frac{1}{2}\left[E_{\alpha, 1}\left(+z^{1 / 2}\right)+E_{\alpha, 1}\left(-z^{1 / 2}\right)\right],
$$

then we can rewrite (20) as follows:

$$
f_{g}(t, x)=\frac{1}{2}\left[u_{g,-\lambda}(t, x)+u_{g, \lambda}(t, x)\right],
$$

where $u_{g, \lambda}$ is given in (18).

So, we can conclude that the general solution of the Cauchy problem (19) for the time fractional diffusion equation turns out to be a linear combination, with coefficients equals to $1 / 2$, of the two general solutions of the Cauchy problems for the fractional Dirac-type equations represented by (17) and by the problem obtained when $\lambda$ is replaced by $-\lambda$ in (17).

In particular, the fundamental solution of (19), when $g(x)=\delta(x)$, turns out to be

$$
f(t, x)=\frac{1}{2 \lambda t^{\alpha}} W\left(-\frac{|x|}{t^{\alpha} \lambda} ;-\alpha, 1-\alpha\right), \quad(0<\alpha<1),
$$

where $W(z ; \alpha, \beta)$ is the Wright special function $([1,18.1(27)])$.

Therefore, if we assume $\lambda=1$ in (19), we obtain

$$
f(t, x)=\frac{1}{2 t^{\alpha}} W\left(-\frac{|x|}{t^{\alpha}} ;-\alpha, 1-\alpha\right)=\frac{1}{2}\left[u_{1}(t, x)+u_{2}(t, x)\right],
$$

where

$$
u_{1}(t, x)= \begin{cases}\frac{1}{t^{\alpha}} W\left(\frac{x}{t^{\alpha}} ;-\alpha, 1-\alpha\right) & x \leq 0 \\ 0 & x>0\end{cases}
$$


and

$$
u_{2}(t, x)= \begin{cases}0 & x<0, \\ \frac{1}{t^{\alpha}} W\left(-\frac{x}{t^{\alpha}} ;-\alpha, 1-\alpha\right) & x \geq 0 .\end{cases}
$$

are the functions appearing in (16), fundamental solutions of (17) when $\lambda=1$ and $\lambda=-1$, respectively (see [3]).

\section{Invariance with respect to Inversions and Transla- tions in time and/or space}

The aim of this section is to establish whether the system (5) turns out to be invariant under inversions and translations in time and/or space, and Galileo transform, and how the non-local property of the time fractional differential operator affects these results.

Let us firstly consider the spatial inversion $P: x^{\prime}=-x$ of the system (5), and set

$$
\psi^{\prime}\left(t, x^{\prime}\right)=S \psi\left(t, x\left(x^{\prime}\right)\right) .
$$

We look for a matrix $S$ so that the transformed multicomponent function $\psi^{\prime}\left(t, x^{\prime}\right)$ is still a solution of the system (5), this is:

$$
A \partial_{t}^{\alpha} \psi^{\prime}\left(t, x^{\prime}\right)+B \partial_{x^{\prime}} \psi^{\prime}\left(t, x^{\prime}\right)=0, \quad \psi^{\prime}\left(t, x^{\prime}\right)=S\left(\begin{array}{l}
u_{1}\left(t, x\left(x^{\prime}\right)\right) \\
u_{2}\left(t, x\left(x^{\prime}\right)\right)
\end{array}\right),
$$

where the fractional derivative $\partial_{t}^{\alpha}$ could be specified in this context either through (7) or (8).

We calculate

$$
\partial_{x^{\prime}} \psi^{\prime}\left(t, x^{\prime}\right)=S \partial_{x^{\prime}} \psi\left(t, x\left(x^{\prime}\right)\right)=-S \partial_{x} \psi(t, x)
$$

and this allows us to write:

$$
A \partial_{t}^{\alpha} \psi^{\prime}\left(t, x^{\prime}\right)+B \partial_{x^{\prime}} \psi^{\prime}\left(t, x^{\prime}\right)=A S \partial_{t}^{\alpha} \psi(t, x)-B S \partial_{x} \psi(t, x) .
$$

Therefore, in order to be (28) verified, it has to turn out

$$
S^{-1} A S \partial_{t}^{\alpha} \psi(t, x)-S^{-1} B S \partial_{x} \psi(t, x)=0,
$$

and, according to (4), when $S=A$, this requirement is fulfilled, being $\psi(t, x)$ solution of (5). Thus, the system of fractional evolution equations (5) is invariant under spatial inversion, as well as the Time Fractional Diffusion Equation (6) with respect to this transformation.

Let us now consider the Time inversion $T: t^{*}=-t$ and set

$$
\psi^{*}\left(t^{*}, x\right)=T \psi\left(t\left(t^{*}\right), x\right) .
$$

Then, as for the spatial inversion, we have to find a matrix $T$ such that $\psi^{*}\left(t^{*}, x\right)$ is solution of the system of equations (5), where now it is necessary to specify the used definition of fractional derivative $\partial_{t}^{\alpha}$. 
If we assume the Caputo fractional derivative (8) in (5), then we have

$$
A_{a}^{C} D_{t^{*}}^{\alpha} \psi^{*}\left(t^{*}, x\right)+B \partial_{x} \psi^{*}\left(t^{*}, x\right)=0, \quad \psi^{*}\left(t^{*}, x\right)=T\left(\begin{array}{c}
u_{1}\left(t\left(t^{*}\right), x\right) \\
u_{2}\left(t\left(t^{*}\right), x\right)
\end{array}\right) .
$$

It results

$$
{ }_{a}^{C} D_{t^{*}}^{\alpha} \psi^{*}\left(t^{*}, x\right)=T(-1)^{\alpha}{ }_{-a}^{C} D_{t}^{\alpha} \psi(t, x) .
$$

and, in particular, when $a=0$ :

$$
{ }_{0}^{C} D_{t^{*}}^{\alpha} \psi^{*}\left(t^{*}, x\right)=T(-1)^{\alpha}{ }_{0}^{C} D_{t}^{\alpha} \psi(t, x) .
$$

Therefore, in general, the left side of the equation in (31) takes the form

$$
(-1)^{\alpha} A T{ }_{-a}^{C} D_{t}^{\alpha} \psi(t, x)+B T \partial_{x} \psi(t, x),
$$

and we only can have invariance of (5), with respect to time inversion, if $a=0$ and the matrix $T$ verifies

$$
\left\{\begin{array}{c}
(-1)^{\alpha} A T=T A \\
B T=T B
\end{array}\right.
$$

The matrix $T=B$ fulfils the above conditions while $(-1)^{\alpha}=-1$, that means

$$
(-1)^{\alpha}=e^{i(\pi+2 n \pi) \alpha}=-1=e^{i(\pi+2 k \pi)},
$$

with $n, k \in\{\mathbb{N} \cup\{0\}\}$, and this implies

$$
\alpha=\frac{1+2 k}{1+2 n}, k=0,1,2, \ldots, \quad n=k+1, k+2, k+3 \ldots,
$$

as the hypothesis $0<\alpha<1$ has to be preserved.

Some particular values of $\alpha$, for which the invariance of the system (5) under time inversion holds, are the followings

$$
\alpha=\frac{1}{3}, \frac{1}{5}, \frac{1}{7}, \ldots, \frac{3}{5}, \frac{3}{7}, \frac{3}{9}, \ldots, \frac{5}{7}, \frac{5}{9}, \frac{5}{11}, \ldots
$$

Note that the Time Fractional Diffusion Equation (6) is invariant under time inversion while $(-1)^{2 \alpha}=1$, that holds and it is well defined for

$$
\alpha=\frac{k}{1+2 n}, n=1,2,3, \ldots, \quad, k=1,2, \ldots, 2 n,
$$

this is,

$$
\alpha=\frac{1}{3}, \frac{2}{3}, \frac{1}{5}, \frac{2}{5}, \frac{3}{5}, \frac{4}{5}, \frac{1}{7}, \frac{2}{7}, \ldots, \frac{6}{7}, \frac{1}{9}, \ldots,
$$

in agreement with known results for the classical one-dimensional Time Diffusion Equation $(\alpha=1 / 2)$.

The invariance properties under Time Inversion of the systems (5) and (6) are a partial characterization of the transition between the parabolic $(\alpha=1 / 2)$ and hyperbolic $(\alpha=1)$ behaviors.

If we repeat the computations when $\partial t^{\alpha}={ }_{a}^{R L} D_{t^{*}}^{\alpha}$ in (5), we obtain the same results so that we can deal in this context with any of the two definitions of fractional derivative without losing generality. 
Now, if we consider the space-time inversion $P T: t^{*}=-t, x^{\prime}=-x$ and we assume

$$
\bar{\psi}\left(t^{*}, x^{\prime}\right)=R \psi\left(t\left(t^{*}\right), x\left(x^{\prime}\right)\right),
$$

over the system of equations (5), repeating exactly the same calculations as above the system turns out to be invariant under space-time inversion if we still restrict our study to the case of $a=0$ in the lower extreme of integration of the fractional derivative and when the matrix $R$ fulfils

$$
\left\{\begin{array}{c}
(-1)^{\alpha} A R=R A \\
B R+R B=0
\end{array}\right.
$$

Therefore it must be distinguished between two cases:

- if $(-1)^{\alpha}=-1$, that means

$$
\alpha=\frac{1+2 k}{1+2 n}, k=0,1,2, \ldots, \quad n=k+1, k+2, k+3 \ldots,
$$

then for $R=A B=-B A$ the system (5) is invariant under space-time inversion.

- if $(-1)^{\alpha}=1$, that means

$$
\alpha=\frac{2 k}{1+2 n}, k=1,2,3, \ldots, \quad n=k, k+1, k+2, \ldots,
$$

then the space-time invariance only holds when $R=A$.

The invariance property of system (5) fails, in general, with respect to Space-Time Translation and Galileo Transform. In fact, if we operate with a space-time translation on the system (5), assuming the changes of variable $t^{*}=t+t_{0}, x^{\prime}=x+x_{0}$, where $t_{0}$ and $x_{0}$ are constants, and setting

$$
\tilde{\psi}\left(t^{*}, x^{\prime}\right)=V \psi\left(t\left(t^{*}\right), x\left(x^{\prime}\right)\right)
$$

the system turns out to be invariant under this space-time transformation just for the trivial case of $t_{0}=0$ and $V=I$. Actually, for the Caputo derivative, for example, it results

$$
{ }_{a}^{C} D_{t^{*}}^{\alpha} \tilde{\psi}\left(t^{*}, x^{\prime}\right)=V{ }_{a-t_{0}}^{C} D_{t}^{\alpha} \psi(t, x) .
$$

In the case of the Galileo Transform: $x^{\prime}=x+v t, t^{\prime}=t$, with $v>0$ constant, we set

$$
\hat{\psi}\left(t, x^{\prime}\right)=W \psi\left(t, x\left(x^{\prime}, t\right)\right)
$$

in (5). In general, we do not have invariance of the system (5) with respect to Galileo Transform, as well as it occurs with the classical Dirac equation. Indeed, it is

$$
{ }_{a}^{C} D_{t}^{\alpha} \hat{\psi}\left(t, x^{\prime}\right)=W{ }_{a}^{C} D_{t}^{\alpha} \psi(t, x)-v W_{a} I_{t}^{1-\alpha} \psi_{x}(t, x),
$$

where $I_{t}^{\alpha}$ is the Riemann-Liouville fractional integral defined as

$$
\left({ }_{a} I_{x}^{\alpha} f\right)(x)=\frac{1}{\Gamma(\alpha)} \int_{a}^{x}(x-t)^{\alpha-1} f(t) d t
$$


with $x>a, \alpha>0, f \in L_{l o c}^{1}(a, \infty)$ and $\left({ }_{a} I_{t}^{0} f\right)(x)=f(x)$.

Then, the transformed multicomponent function $\hat{\psi}\left(t, x^{\prime}\right)$ is still a solution of (5) if the system

$$
\begin{gathered}
A_{a}^{C} D_{t}^{\alpha} \hat{\psi}\left(t, x^{\prime}\right)+B \partial_{x^{\prime}} \hat{\psi}\left(t, x^{\prime}\right)= \\
=A W_{a}^{C} D_{t}^{\alpha} \psi(t, x)-v A W_{a} I_{t}^{1-\alpha} \psi_{x}(t, x)+B W \partial_{x} \psi(t, x),
\end{gathered}
$$

is equal to the zero vector, that implies

$$
A W_{a} I_{t}^{1-\alpha} \psi_{x}(t, x)=0 .
$$

Therefore, in general, we do not have invariance of the system (5) with respect to Galileo Transform because, in view of the evaluation of the beta integral [1], valid for all $p>0$ and $q>0$ (or $\operatorname{Re}(p)>0$ and $\operatorname{Re}(q)>0$ ), namely

$$
B(p, q):=\int_{0}^{1} u^{p-1}(1-u)^{q-1} d u=\frac{\Gamma(p) \Gamma(q)}{\Gamma(p+q)},
$$

it can be proved the following property for the Riemann-Liouville fractional integral

$$
{ }_{a} I_{t}^{\alpha}(t-a)^{\beta}=\frac{\Gamma(\beta+1)}{\Gamma(\beta+\alpha+1)}(t-a)^{\beta+\alpha}
$$

when $\beta>-1$. As a consequence, it results ${ }_{a} I_{t}^{1-\alpha} \psi_{x}(t, x)=0$ just in case $\psi_{x}(t, x)=0$ for any $0<\alpha<1$, that means $\psi(t, x)=\psi(t)$, constant function in $x$. If this condition is not fulfilled, as it occurs in general, then, being $A=\left(\begin{array}{ll}0 & 1 \\ 1 & 0\end{array}\right)$ in our case, we can obtain a restricted class of functions $u_{1}$ and $u_{2}$ for which (5) is invariant under Galileo transform.

$$
\begin{array}{r}
\text { Given } W=\left(\begin{array}{ll}
w_{11} & w_{12} \\
w_{21} & w_{22}
\end{array}\right) \text {, the system (38) can be reduced to } \\
\left\{\begin{array}{l}
w_{21} I_{t}^{1-\alpha} \frac{\partial}{\partial x} u_{1}(t, x)+w_{22} I_{t}^{1-\alpha} \frac{\partial}{\partial x} u_{2}(t, x)=0 \\
w_{11} I_{t}^{1-\alpha} \frac{\partial}{\partial x} u_{1}(t, x)+w_{12} I_{t}^{1-\alpha} \frac{\partial}{\partial x} u_{1}(t, x)=0
\end{array}\right.
\end{array}
$$

and, if $\operatorname{det}(W)=0$, that means $w_{12} w_{21}-w_{11} w_{22}=0$, then it has the solution $\frac{\partial}{\partial x} u_{1}(t, x)=$ $-\frac{w_{22}}{w_{21}} \frac{\partial}{\partial x} u_{2}(t, x)$ that implies $u_{1}(t, x)=-\frac{w_{22}}{w_{21}} u_{2}(t, x)+c(t)$, where $c(t)$ is a constant function in $x$.

The general non invariance of the fractional evolution equations under Time Translation and Galileo transform is due to the non-local property of the time fractional derivative and it is in contrast with the invariance results holding for the Dirac equation, with respect to the Time Translation, as a fundamental requirement to be the relativity principle verified [19].

\section{A Conserved Quantity: the Fractional Hamiltonian}

The invalidity of the invariance of the fractional Dirac-type system (5) under Time Translation does not prevent it from possessing a fractional conserved quantity, analogous of the Hamiltonian for the classical Dirac system. 
It is well known (see, for example, [19]) that the Lagrangian density for the classical Dirac equation obtained from (5) when $\alpha=1$, is given by

$$
\mathcal{L}(t, x)=\bar{\psi} A \partial_{t} \psi+\bar{\psi} B \partial_{x} \psi
$$

with $\psi=\psi(t, x)=\left(u_{1}(t, x), u_{2}(t, x)\right)^{T}, \bar{\psi}=\bar{\psi}(t, x)=\psi^{+} A$, and $\psi^{+}=\psi^{+}(t, x)=$ $\left(u_{1}^{*}(t, x), u_{2}^{*}(t, x)\right)$, complex conjugated of $\psi$ that verifies the conjugated equation of (5) with $\alpha=1$ :

$$
\partial_{t} \psi^{+} A^{+}+\partial_{x} \psi^{+} B^{+}=0 .
$$

Therefore, the Hamiltonian density will be

$$
\mathcal{H}(t, x)=\frac{\partial \mathcal{L}(t, x)}{\partial\left(\partial_{t} \psi\right)} \partial_{t} \psi-\mathcal{L}(t, x)=\bar{\psi} A \partial_{t} \psi-\mathcal{L}(t, x)=-\psi^{+} C \partial_{x} \psi
$$

with $C=A B=-B A$, the Hamiltonian

$$
H(t, x)=\int_{-\infty}^{+\infty} \mathcal{H}(t, x) d x=-\int_{-\infty}^{+\infty} \psi^{+} C \partial_{x} \psi d x
$$

and, its time derivative,

$$
\frac{d}{d t} H(t, x)=\int_{-\infty}^{+\infty} \partial_{x}\left[\psi^{+} \partial_{x} \psi\right] d x
$$

provided that we restrict ourselves to the pure real matrices $A$ and $B$ so that the equivalence $\partial_{t} \psi^{+}=-\partial_{x} \psi^{+} C$ is verified. In this case, if we assume, for example, the initial condition $\psi^{+} \partial_{x} \psi \rightarrow 0$ when $|x| \rightarrow \infty$, we can conclude that there exists a conserved quantity associated to equation (5) with $\alpha=1$, given by the Hamiltonian (41).

In what follows, we want to show that, as well as we did above, it is possible to find a conserved quantity associated to the system of fractional Dirac-type equations (5) for general $0<\alpha<1$, even if it does not present invariance with respect to time translation.

We start defining, by analogy with the classical Dirac case, a formal "fractional Lagrangian density" related to (5)

$$
\mathcal{L}^{\alpha}(t, x)=\bar{\psi} A \partial_{t}^{\alpha} \psi+\bar{\psi} B \partial_{x} \psi
$$

and a formal "fractional Hamiltonian density"

$$
\mathcal{H}_{\alpha}(t, x)=\frac{\partial \mathcal{L}^{\alpha}(t, x)}{\partial\left(\partial_{t}^{\alpha} \psi\right)} \partial_{t}^{\alpha} \psi-\mathcal{L}^{\alpha}(t, x)=\bar{\psi} A \partial_{t}^{\alpha} \psi-\mathcal{L}^{\alpha}(t, x)=-\psi^{+} C \partial_{x} \psi
$$

The final expression in (44) is equivalent to (40) and can be simplified observing that, being $A$ and $B$ pure real matrices, if $\psi$ is a solution of (5), then also $\psi^{+}$has to solve it, reason why a pure real solution of (5) can always be found. Therefore, we will assume that $\psi$ it is a pure real solution of (5) and this allows us to write the "fractional Hamiltonian" as

$$
H_{\alpha}(t, x)=\int_{-\infty}^{+\infty} \mathcal{H}_{\alpha}(t, x) d x=-\int_{-\infty}^{+\infty} \psi^{T} C \partial_{x} \psi d x
$$


and, consequently,

$$
\frac{d}{d t} H_{\alpha}(t, x)=-\int_{-\infty}^{+\infty}\left[\partial_{t} \psi^{T} C \partial_{x} \psi+\psi^{T} C \partial_{x} \partial_{t} \psi\right] d x=-\int_{-\infty}^{+\infty} \partial_{x}\left[\psi^{T} C \partial_{t} \psi\right] d x .
$$

It has been used the equivalence $\partial_{t} \psi^{T} C \partial_{x} \psi=\partial_{x} \psi^{T} C \partial_{t} \psi$, due to the fact that the matrix $C=A B$, with pure real $A$ and $B$, only can be of two types:

$$
C_{1}=\left(\begin{array}{cc}
c_{11} & 0 \\
0 & -c_{11}
\end{array}\right) \quad \text { o } \quad C_{2}=\left(\begin{array}{cc}
0 & c_{12} \\
c_{12} & 0
\end{array}\right)
$$

where $c_{11}$ and $c_{12}$ take the values \pm 1 .

At this point it is necessary to specify the definition of the fractional derivative in use.

When $0<\alpha<1$ the Riemann-Liouville fractional derivative $\partial_{t}{ }^{\alpha}={ }^{R L} D_{t}^{\alpha}$, according to (9), fulfils the following identity

$$
\left({ }^{R L} D_{t}^{\alpha} \psi\right)(t, x)=\left(I_{t}^{1-\alpha} \partial_{t} \psi\right)(t, x)+\frac{t^{-\alpha}}{\Gamma(1-\alpha)} \psi(0, x),
$$

being $\left({ }^{R L} D_{t}^{\alpha} \psi(0, x)\right)(t, x)=\frac{t^{-\alpha}}{\Gamma(1-\alpha)} \psi(0, x)$ and $\left({ }^{C} D_{t}^{\alpha} \psi\right)(t, x)=\left(I_{t}^{1-\alpha} \partial_{t} \psi\right)(t, x)$ by definition (8).

Now, if we introduce the Riemann-Liouville fractional derivative in (5), using the fact that this derivative is the left inverse operator of the Riemann-Liouville fractional integral, we can write

$$
\begin{gathered}
\left(\partial_{t} \psi\right)(t, x)=-C \partial_{x}\left({ }^{R L} D_{t}^{1-\alpha} \psi\right)(t, x)-A\left({ }^{R L} D_{t}^{1-\alpha} \frac{t^{-\alpha}}{\Gamma(1-\alpha)} \psi(0, x)\right)(t, x)= \\
=-C \partial_{x}\left({ }^{R L} D_{t}^{1-\alpha} \psi\right)(t, x)-A \partial_{t} \psi(0, x)=-C \partial_{x}\left({ }^{R L} D_{t}^{1-\alpha} \psi\right)(t, x) .
\end{gathered}
$$

In a similar straightforward way it can be proved that result (48) holds exactly the same when the Caputo derivative appears in (5).

Therefore, when $\alpha=1 / 2$ both derivatives verify

$$
\left(\partial_{t} \psi\right)(t, x)=C^{2}\left(\partial_{x}^{2} \psi\right)(t, x)=\left(\partial_{x}^{2} \psi\right)(t, x) .
$$

In agreement with (48), the expression for the Hamiltonian time derivative (46) takes the form:

$$
\frac{d}{d t} H_{\alpha}(t, x)=-\int_{-\infty}^{+\infty} \partial_{x}\left[\psi^{T} C \partial_{t} \psi\right] d x=\int_{-\infty}^{+\infty} \partial_{x}\left[\psi^{T} \partial_{x}^{R L} D_{t}^{1-\alpha} \psi\right]
$$

when $0<\alpha<1$ and, in particular,

$$
\frac{d}{d t} H_{1 / 2}(t, x)=\int_{-\infty}^{+\infty}-\partial_{x}\left[\psi^{T} C \partial_{x}^{2} \psi\right] d x
$$


for $\alpha=1 / 2$, when the fractional derivative is either of the Riemann-Liouville or of the Caputo type.

Therefore, we can conclude that, when $0<\alpha<1$, if the condition

$$
\begin{gathered}
\frac{d}{d t} H_{\alpha}(t, x)=\int_{-\infty}^{+\infty} \partial_{x}\left[\psi^{T} \partial_{x}{ }^{R L} D_{t}^{1-\alpha} \psi\right] d x= \\
=\left.\left[u_{1} \partial_{x}{ }^{R L} D_{t}^{1-\alpha} u_{1}+u_{2} \partial_{x}{ }^{R L} D_{t}^{1-\alpha} u_{2}\right]\right|_{x=-\infty} ^{x=+\infty}=0,
\end{gathered}
$$

is fulfilled, then a conserved quantity exists associated to equation (5) and it is given by the fractional Hamiltonian (45).

For the particular case of $\alpha=1 / 2$, we deduce from (51) an alternative condition, equivalent to (52), to provide the existence of the conserved quantity $H_{1 / 2}(t, x)$ :

$$
\frac{d}{d t} H_{1 / 2}=\int_{-\infty}^{+\infty}-\partial_{x}\left[\psi^{T} C \partial_{x}^{2} \psi\right] d x=-\left.\psi^{T} C \partial_{x}^{2} \psi\right|_{x=-\infty} ^{x=+\infty}=0
$$

that means

$$
\left.\left(u_{1} \partial_{x}^{2} u_{1}-u_{2} \partial_{x}^{2} u_{2}\right)\right|_{x=-\infty} ^{x=+\infty}=0
$$

when $C=C_{1}$, and

$$
\left.\left(u_{1} \partial_{x}^{2} u_{2}+u_{2} \partial_{x}^{2} u_{1}\right)\right|_{x=-\infty} ^{x=+\infty}=0,
$$

when $C=C_{2}$. Both conditions (53) and (54) come true when, for example, $\left|u_{h}\right| \rightarrow 0$ and $\partial_{x}^{2} u_{h}$ is bounded when $|x| \rightarrow \infty$, for $h=1,2$.

We can conclude this section recalling some results obtained in [3].

If we consider the system of fractional equations of Dirac-type (5) when $\partial_{t}{ }^{\alpha}={ }^{C} D_{t}^{\alpha}$, when $A$ and $B$ are given by (15) so that it turns out to be

$$
C=C_{1}=\left(\begin{array}{cc}
-1 & 0 \\
0 & 1
\end{array}\right)
$$

and we complete (5) with the initial conditions

$$
\lim _{|x| \rightarrow \infty} \psi(t, x)=0, \quad \psi(0+, x)=\delta(x),
$$

then the localized fundamental solutions are given by (25) y (26).

In [5] the asymptotic behavior of the Wright function $W(z ; \alpha, \beta)$, for the case of $\alpha=-\nu$ and $\beta=1-\nu$, has been studied. In particular, if the function $M(z ; \nu)=$ $W(-z ;-\nu, 1-\nu)$ is introduced, when the argument $z=r>0$ is real and positive and $r \rightarrow+\infty$, it holds

$$
M\left(\frac{r}{\nu} ; \nu\right) \sim a(\nu) r^{(\nu-1 / 2) /(1-\nu)} \exp \left[-b(\nu) r^{1 /(1-\nu)}\right],
$$

where $a(\nu)=1 / \sqrt{2 \pi(1-\nu)}, b(\nu)=(1-\nu) / \nu$.

This implies an asymptotic exponential decay of our solutions $u_{1} \mathrm{y} u_{2}$, and, consequently, that $\left|u_{h}\right| \rightarrow 0$ when $|x| \rightarrow \infty$ for $h=1,2$. 
In order to ensure the existence of the conserved quantity (45) when $0<\alpha<1$, we also have to analyze the asymptotic behavior of $\partial_{x}{ }^{R L} D_{t}^{1-\alpha} u_{h}$ for $h=1,2$.

It turns out to be:

$$
\begin{gathered}
{ }^{R L} D_{t}^{1-\alpha}\left(\frac{1}{t^{\alpha}} W\left(-\frac{|x|}{t^{\alpha}} ;-\alpha, 1-\alpha\right)\right)=\partial_{t} I_{t}^{\alpha}\left[\sum_{k=0}^{+\infty} \frac{-(|x|)^{k} t^{-\alpha k-\alpha}}{k ! \Gamma(-\alpha k+1-\alpha)}\right]= \\
=\partial_{t}\left[\sum_{k=0}^{+\infty} \frac{-(|x|)^{k} t^{-\alpha k}}{k ! \Gamma(1-\alpha k)}\right]=\left[\sum_{k=0}^{+\infty} \frac{-(|x|)^{k} t^{-\alpha k-1}}{k ! \Gamma(-\alpha k)}\right]= \\
=\frac{1}{t} W\left(-\frac{|x|}{t^{\alpha}} ;-\alpha, 0\right)=\frac{\alpha|x|}{t^{\alpha+1}} W\left(-\frac{|x|}{t^{\alpha}} ;-\alpha, 1-\alpha\right)
\end{gathered}
$$

if we use the property $W(-z ;-\nu, 1-\nu)=\frac{1}{\nu z} W(-z ;-\nu, 0)$; therefore, (55) implies that ${ }^{R L} D_{t}^{1-\alpha} u_{h}$, as well as $\partial_{x}{ }^{R L} D_{t}^{1-\alpha} u_{h}$, will also decay exponentially when $|x| \rightarrow \infty$ for $h=1,2$.

\section{Conclusions}

We have treated a generalization of the classical free Dirac equations, namely the fractional evolution equations of Dirac-type. For the localized solutions of these equations we have derived their relation with the corresponding solution of the fractional diffusion equation, showing how the latter turns out to be a linear combination of the formers, as well as the D'Alembert solution of the classical wave equation is a linear combination of the solutions of the first order equations derived from the decomposition of the second order wave operator into its corresponding square-root operators.

Following the analogy existing between the fractional evolution equations and the classical Dirac equation, we have studied their internal symmetries with respect to certain transformations in space and/or time. The system of fractional Dirac-type equations is invariant under Spatial Inversion for each $0<\alpha<1$, whereas it possesses invariance under Time Inversion only for certain values of the fractional index $\alpha$, so inclosing the hyperbolic behavior of the classical Dirac equation or of the Time Fractional Diffusion equation (6) when $\frac{1}{2}<\alpha \leq 1$ (including the classical wave equation corresponding to $\alpha=1$ ), and the parabolic one of the Time Fractional Diffusion equation (6) when $0<\alpha \leq \frac{1}{2}$ (including the classical diffusion equation corresponding to $\alpha=1 / 2$ ). In keeping with the joint Space-Time Inversion, a range of validity for the invariance is located, still depending on the index $\alpha$, but wilder then the which one corresponding to the only Time Inversion.

The system proves to be never invariant under Time Translation and Galileo transform, due to the non-local property of the time fractional derivative. This lack of invariance of the fractional evolution equations under time translation, is in contrast with the invariance results holding for the classical Dirac equation, with respect to the same transformation, as a fundamental requirement to be the relativity principle verified, but does not prevent the fractional Dirac-type system from possessing a fractional conserved quantity, analogous of the Hamiltonian for the classical Dirac system. 


\section{Acknowledgements}

T.P. and L.V. thank the partial support of the Ministerio de Ciencia y Tecnologa of Spain under grant BFM 2002-02359. T.P. acknowledges the predoctoral fellowship of the Programa de Formación de Personal Investigador de la Comunidad de Madrid (5793/2002)

\section{References}

[1] Erdelyi A., Magnus W., Oberhettinger F. and Tricomi F., G. Higher Transcendental Functions, Vol.3, McGraw-Hill Book Corp., New York, 1953.

[2] Hilfer R., Applications of Fractional Calculus in Physics. World Scientific, 2000.

[3] Kilbas A.A., Pierantozzi T., Trujillo J. and Vázquez L., On the solution of fractional evolution equations. Journal of Physics A: Mathematical and General, 37, 2004, 3271-3283.

[4] Mainardi F., Paradisi P. and Gorenflo R., Probability distributions generated by fractional diffusion equations, Econophysics: an Emerging Science. Kertesz J. and Kondor I. eds., Dordrecht: Kluwer, 1999.

[5] Mainardi F. and Tomirotti M., On a special function arising in the time fractional diffusionwave equation, Transform Methods and Special Functions, Sofia 1994. Rusev P., Dimovski I. and Kiryakova V. eds., Science Culture Technology, Singapore, 1995, 171-183.

[6] Mainardi F., On the initial value problem for the fractional diffusion-wave equation, Waves and Stability in Continuous Media, Bologna, 1993, Ser. Adv. Math. Appl. Sci., n. 23, 1994, 246-251.

[7] Metzler R. and Klafter J., The Random Walk's Guide to Anomalous Diffusion: A Fractional Dynamics Approach, Phys. Reports, 339, 2000, 1-77.

[8] Metzler R. and Klafter J., Accelerating Brownian motion: a fractional dynamics approach to fast diffusion, Europhys. Lett., 51, 2000, 492-498.

[9] Metzler R. and Nonnemacher T.F., Space- and Time-fractional diffusion and wave equations, fractional Fokker-Planck equations, and physical motivation, Chem. Phys., 284, 2002, n. $1-2,67-90$.

[10] Miller S. K. and Ross B., An introduction to the Fractional Calculus and Fractional Differential Equations, John Wiley \& Sons Inc., New York, 1993.

[11] Morinaga K. and Nono T., J. Sci. Hiroshima Univ. (A), 16, 1952, 13-41.

[12] Oldham K. B. and Spanier J., The replacement of Fick's laws by a formulation involving semidifferentiation, J. Electroanalyt. Chem., 26, 1970, 331-341.

[13] Oldham K. B. and Spanier J., The Fractional Calculus, Academic Press, New York-London, 1974.

[14] Podlubny I., Fractional Differential Equations, Mathematics in Science and Engineering. 198 Academic Press, San-Diego, 1999.

[15] Ross B., Fractional Calculus and its Applications, Lecture Notes in Mathematics, 457. Springer-Verlag, 1975.

[16] Saichev A. I. and Zaslavsky G. M., Fractional kinetic equations: solutions and applications, Chaos, 7, 1997, 753-764.

[17] Samko S. G., Kilbas A.A., Marichev O. I., Fractional Integrals and Derivatives. Theory and Applications, Gordon and Breach, Yverdon, 1993. 
[18] Schneider W. R. and Wyss W., Fractional diffusion and wave equations, J. Math. Phys., 30, 1989, 134-144.

[19] Schweber S. S., An Introduction to Relativistic Quantum Field Theory, Harper Int. Ed. New York, 1966.

[20] Sokolov I., Klafter J. and Blumen A., Fractional Kinetics, Physics Today, 55, 2002,48-54.

[21] Vázquez L., Fractional diffusion equation with internal degrees of freedom, J. Comp. Math., Vol. 21, n. 4, 2003, 491-494.

[22] Vázquez L. and Vilela Mendes, R., Fractionally coupled solutions of the diffusion equation, Appl. Math. Comp., 141, 2003, 125-130.

[23] Vlad M. O., Metzler R., Nonnenmacher T. F. and Mackey M. C., Universality classes for asymptotic behavior of relaxation processes in systems with dynamical disorder: Dynamical generalizations of stretched exponential, J. Math. Phys., 37, 1996, 2279-2306. 